\title{
SIZE DISTRIBUTION ANALYSES FOR ESTIMATING DIAMOND GRADE AND VALUE
}

\author{
Chapman JG and Boxer GL \\ Independent consultants, Perth, Western Australia
}

\section{INTRODUCTION}

Grade assessment is a key component of any new diamond resource evaluation. Depending on the degree of deposit development, these assessments can be based on either micro diamonds from drill samples or macro diamonds from bulk samples. Clearly the bulk samples provide more useful data, as not only are the diamonds more representative of a production scenario, but also they enable a quality assessment for price estimates. The analysis of micro diamond data is usually the basis upon which decisions are made to proceed to bulk sampling. A full understanding of the relationship between micro and macro diamond populations, coupled with an appreciation for the errors involved in any analysis will ensure that potentially economic deposits are not overlooked and that unnecessary expenditure is not directed at uneconomic deposits.

Factors that are relevant to these deposit assessments include: the relationship between micro and macro distributions, sample size, classification technique (sieving regime, weights), and method of frequency calculation. Some of these aspects are addressed in this paper and apply to primary deposits with limited relevance to alluvial deposits.

With the recovery of significant quantities of macro diamonds from bulk sampling, there is an opportunity to both assign a value to the diamonds and to better describe the size population, enabling predictions of occurrences for large diamonds and plant recovery performance. Commonly the $\$ / \mathrm{ct}$ average price is determined by merely calculating the quotient of the diamond value and the carats recovered, however this approach ignores any statistical understanding of the size populations and values.

The first topic to address is the relationship between micro and macro size frequency distributions (SFD). In this paper, unless specified otherwise, the terms 'macro' and 'micro' are loosely used to refer, respectively, to those sizes recovered with pilot or production plants and those recovered in a laboratory. Where micro:macro ratios are discussed, the size transition is $0.5 \mathrm{~mm}$.

\section{SIZE FREQUENCY DISTRIBUTION}

Frequency is determined by the quantity, $\mathrm{N}$, of

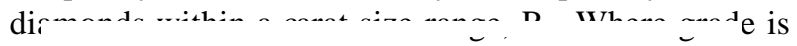
If interest, the quantity of diamonds can be expressed $\mathrm{n}$ per ton terms. Thus;

Frequency $=\mathrm{N} / \mathrm{W} / \mathrm{R}$

where $\mathrm{W}$ is the weight of the host rock sample.

$\mathrm{t}$ is common practice to size diamonds using screens ather than weights. In this instance it is necessary to tpply upper and lower carat weights to each size raction. These weights can be assigned using an ntuitive expression which assumes a shape profile that :xtends across all sizes. This expression is;

$$
\text { Weight }=\mathrm{k} \times(\text { aperture size })^{3}
$$

Where $\mathrm{k}$ is a constant determined so as to yield an appropriate mean stone size (MSS) for an upper and lower screen size. (MSS $\approx 0.9 \times$ average of upper and lower weights).

Because of the wide range of sizes involved in a typical analysis, ranging over a few orders of magnitude, it is useful to present frequency figures logarithmically. The frequency can be plotted against the independent variable of MSS for each size fraction, which is also best presented logarithmically. While it is common to plot SFDs as a cumulative curve, the authors believe such curves do not allow ready interpretation of the distribution and identification of anomalies.

\section{MICRO-MACRO RELATIONSHIP}

Examination of numerous deposits reveal that the general relationship of micro and macro SFDs is as shown in figure 1.

There are four notable features in this relationship:

- The micro frequency distribution is linear.

- The macro frequency distribution conforms to a $2^{\text {nd }}$ order polynomial

- Recovery losses are present in the smaller fractions of both the micro and macro populations. 
- There is a transitional MSS between the linear and polynomial distribution.

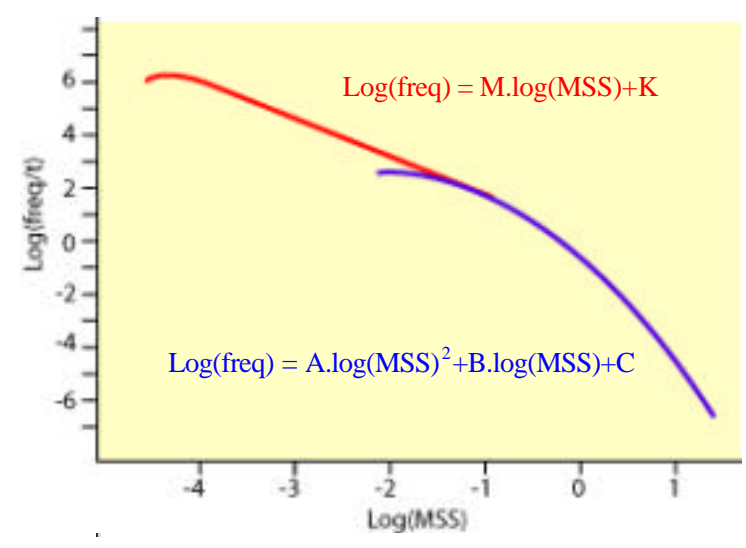

Figure 1: Typical frequency curves for small and large size fractions of diamonds from the same deposit.

The frequency discrepancy between overlap regions (of MSS) of the two sizes is due to the recovery methods involved. For example, micro diamonds extracted by acid digestion will be more efficient than the same size macro diamonds recovered by an HMS plant. The discrepancy can be significant, and it is not uncommon to record an order of magnitude difference at $0.05 \mathrm{ct}$ size!

It is difficult to know the extent to which the micro diamonds recovered from host rock are representative of the host rock from which larger diamonds are recovered. Sometimes there is a frequency mismatch between the two regions. Under these circumstances it is assumed the micro diamonds are from a region having a grade different to that of the bulk sample host rock. However there are suggestions that the macro curve passes above the extrapolation of the micro line, in which case it could be interpreted that breakages of larger stones have occurred, increasing the quantity of a smaller sized fraction.

It is interesting to speculate why the size frequency distribution has two regions, and why the linear relationship does not extend to the large sizes? One might also wonder what the lower size limit is for micro diamonds.

The key issue about the micro-macro relationship is whether their distributions are uniquely interrelated and to what extent the macro relationship can be predicted from the micro distribution. The relevant parameters of the macro frequency distribution that relate to grade are:

- the point at which it departs from the linear (micro) relationship
- the degree of curvature. (A and B coefficients of the quadratic expression)

- the 'height' of the curve. (C coefficient of the quadratic expression)

It has been found that for two deposits having very similar micro distributions, the first two parameters above can be quite different. So an explicit grade determination cannot be made purely from a micro distribution, however if the size distribution is known (for example from the same deposit) the grade can be estimated. An assessment can be made on whether the grade warrants further workings. In the absence of any other measurements, the analysis of micro size frequency distributions can be useful and with analysis of further deposits it is hoped that factors will be found that enable a more accurate estimate of macro diamond distributions and grade.

Micro diamond results are commonly expressed by two figures; the quantity of diamonds recovered for a measured host rock weight and the ratio of the quantity above and below $0.5 \mathrm{~mm}$. The interpretation of these figures is paramount and is not explicitly provided in reports, with different analysts using different criteria to assess the results

\section{DIAMOND COUNTS}

Neither the total number of micro-diamonds recovered from a host rock sample nor the ratio of micro to macro diamonds are useful in isolation. The reason is best illustrated in figure 2, which shows two distributions associated with a high and a low grade deposit. While one line exhibits a higher stone count, it is in fact associated with a lower grade by virtue of the micro:macro ratio.

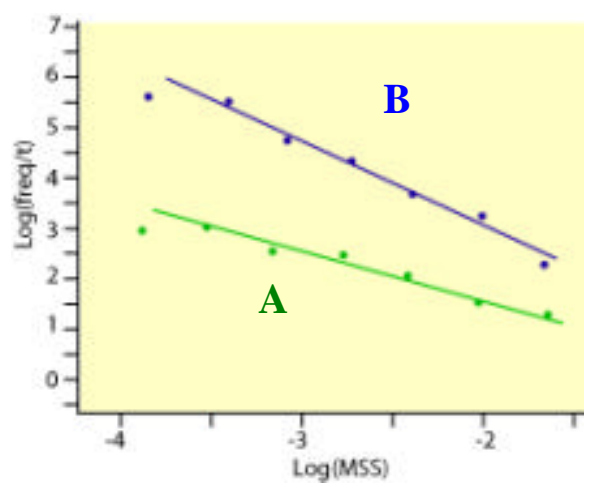

Figure 2: Micro diamond distributions for two deposits. Although having a higher stone count, deposit A has a higher grade than deposit B 
If it is accepted that micro:macro ratios are significant, then it is important to realise the statistical uncertainties in any figure derived. An appreciation of the impact of low stone counts is shown in Figure 3, where the expected micro:macro ratio has been simulated for a particular distribution (described by $\log ($ freq $)=0.8-$ $1.6 \log (\mathrm{MSS}))$.

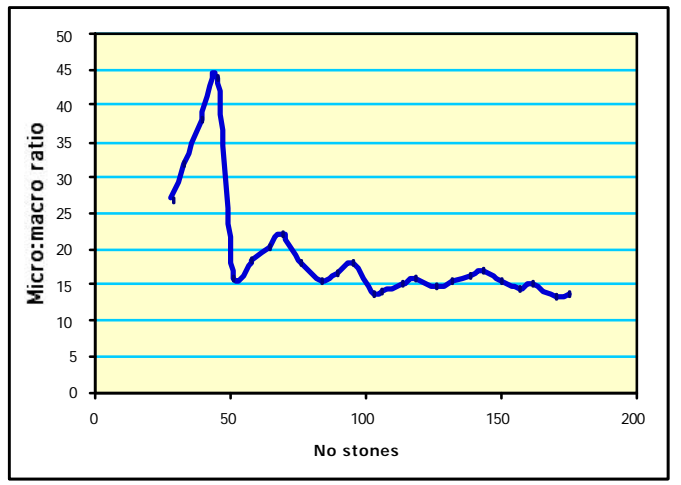

Figure 3: The effect of stone quantity on micro:macro ratios for a particular distribution.

The oscillations occur where the quantity of macro diamonds is low and so each additional stone makes a significant change in the ratio. In order to remove the errors from this traditional approach, a better parameter is to either express the ratio indirectly as the gradient $M$ of the size distribution or to derive a corrected ratio. A corrected ratio can be derived from the frequency distribution, which also enables application of confidence levels (CL) and correction of data relating to recovery losses. Figure 4 shows an example of a micro diamond frequency curve and the associated macro:micro ratio.

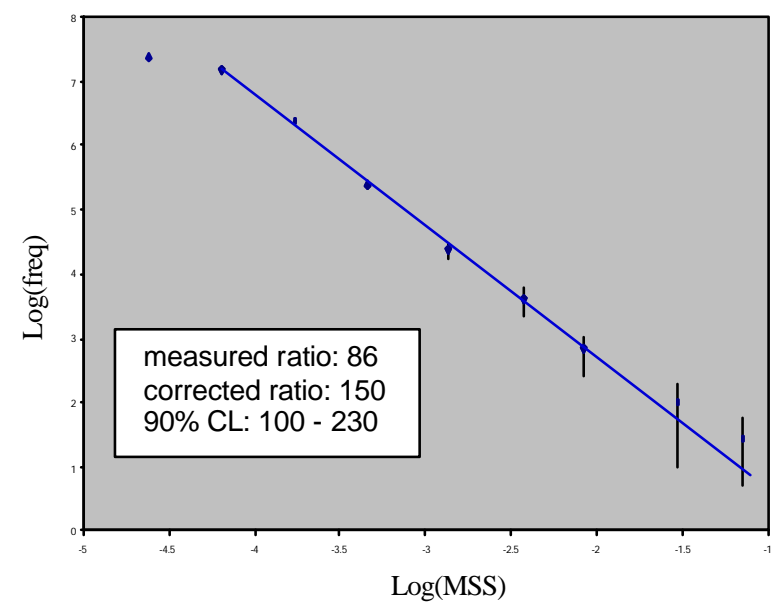

Figure 4: A display of a (real) micro-diamond distribution including statistical errors.
The benefit of a frequency relationship expressed by two parameters defining a line is that the second parameter corresponds to the quantity of diamonds usually reported in an analysis. Using more complex statistical techniques, the standard error for the coefficients can be calculated, which can then be translated into standard errors for the micro:macro ratio.

With a suitably expansive collection of data it may be conceivable to generate criteria linking the linear coefficients, $\mathrm{M}$ and $\mathrm{K}$, for the micro SFD and the likely grade, as shown in figure 5 .

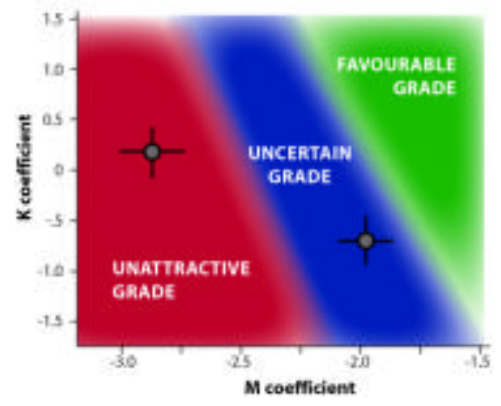

Figure 5: Illustrative grade criteria map for micro SFD expressed as $\log ($ freq $)=M \times \log (\mathrm{MSS})+\mathrm{K}$. Two fictitious example points are shown with error bars.

The inclusion of further parameters may provide better estimates of grade, such as; the MSS within sieve sizes, discriminating between broken and single crystals, and using geological indicators.

\section{MACRO DIAMOND POPULATIONS}

Where the recovery of diamonds is sufficient to yield appreciable quantities of macro diamonds, there is scope to generate far more accurate size frequency distributions and grade calculations. One aspect which this paper does not cover is the variability of grade within a deposit.

As mentioned earlier, the macro diamond SFD has a quadratic nature, and for a single population can be expressed as:

$$
\log (\text { freq })=A \log _{10}(\mathrm{MSS})^{2}+\mathrm{B} \log _{10}(\mathrm{MSS})+\mathrm{C}
$$

Where $\mathrm{A}, \mathrm{B}$ and $\mathrm{C}$ are constants (quadratic coefficients).

A few hundred carats of size greater than about $0.02 \mathrm{ct}$ (+3 DTC sieve) is sufficient to provide sufficient data for a grade determination with an uncertainty of less 
than $10 \%$. Figure 6 shows a plot for a 500 carat sample from a bulk sample. The smallest size has been omitted for the trendline, as it clearly represents a recovery loss. The uncertainties (error bars) are based on $95 \%$ confidence limits using Poisson distributions. Note the low uncertainty in frequencies of the smaller sizes

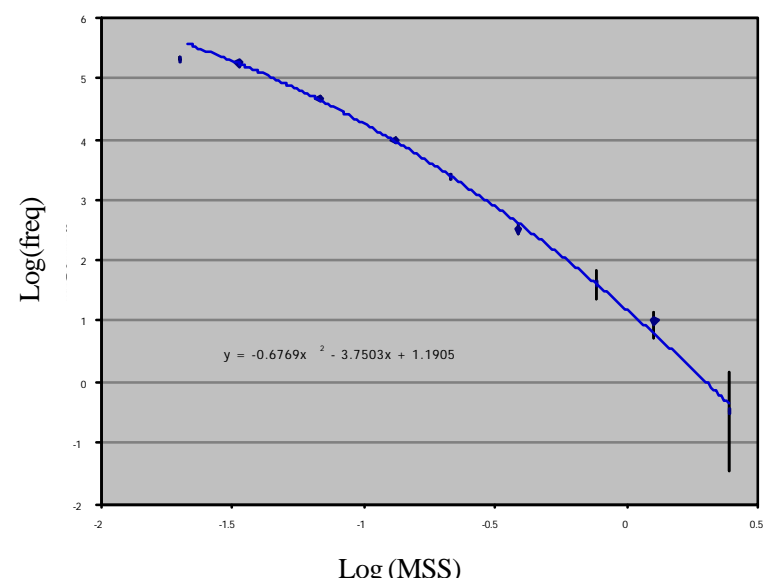

Figure 6: A quadratic line fitted to the frequency curve of 500 carats from a bulk sample.

With algebraic manipulation the quadratic curve can be transformed to a linear relationship enabling uncertainty figures to be assigned to the quadratic coefficients, and hence confidence limits to any extrapolations.

\section{AVERAGE PRICE}

By combining the size distribution with valuation data it is possible to gain a better estimate of the average price for the diamonds in the deposit. This exercise requires a relationship between diamond price and diamond size. A valuer can provide estimates of the price for different size categories, however these estimates have statistical limitations from low diamond counts in larger sizes. Yet, these larger sizes can contribute significantly to the overall price.

The effect of size categories having low diamond counts is that generally the valuation for those categories will be less than that obtained in production. This discrepancy is because the price frequency distribution for a size category is significantly skewed, with only a small proportion of high value diamonds raising the average price substantially. It is not unusual for $80 \%$ of the diamonds in a size class to have a value below the average value for that size. In these instances, it will be appreciated that for low stone counts, say less than 10, there is a high likelihood of only the sub-average value diamonds being represented.
Plotting the average value of each size class as a function of the average weight for each class will show representational errors. For large volumes of diamonds, the value-size relationship plotted logarithmically is generally linear, as seen in figure 7 . On closer inspection, there are two linear regions for this deposit, however in the absence of sufficient quantities of diamonds, reduction to a single line is useful for estimation purposes.

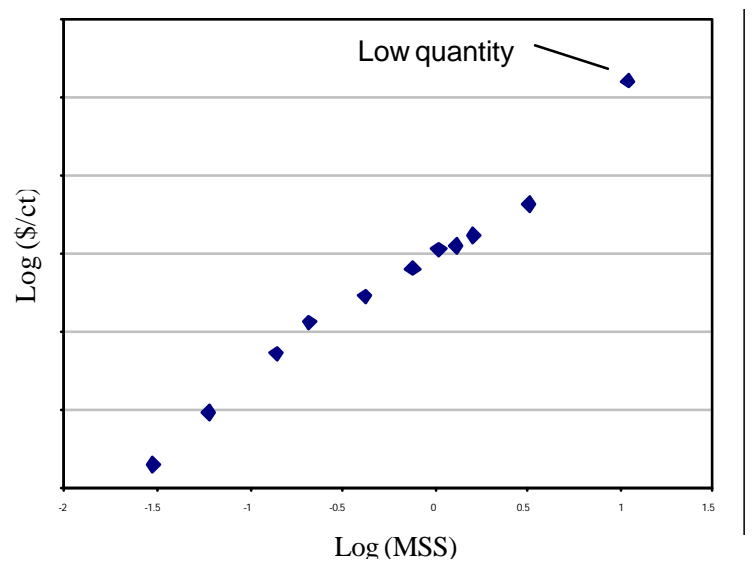

Figure 7: The relationship between price and diamond size for a particular commercial deposit.

Having derived a relationship between size and price, it is possible to assign corrected prices to those supplied by a valuer. Applying these prices to a frequency distribution enables an expected production scenario to be modelled, thus providing a more accurate estimate of the overall \$/ct price for a deposit.

\section{CONCLUSIONS}

The size frequency curve for diamonds ranging from micro to macro sizes comprises both a linear portion and a quadratic portion. Total diamond counts and the ratio of micro to macro diamonds as an indicator of grade can be misleading as the values are very dependent on the volume of diamonds recovered and the efficiency of recovery for the smallest sizes.

It is believed that an explicit method of determining the grade of commercial (macro) diamonds on the basis of the micro diamonds is not achievable, but useful indications of the grade can be gained from the micro size frequency distribution and the coefficients defining its linear relationship. The approach of using distribution plots combined with other morphological or geological parameters may offer a more accurate indicator of grade. 
The macro diamond frequency distribution conforms well to a quadratic relationship, allowing extrapolation to large diamond sizes. Expected average prices for large diamonds can be gained from extrapolations of price - size relationships, which when combined with size frequency expressions provide more accurate estimates of average diamond price than that of only the recovered diamonds.

There are no particular quantities or volumes of diamonds that must be recovered in order to estimate grade or value, however the larger the quantities or volumes involved, the lower the uncertainty of any derived values. It is therefore important that any derived values for say micro:macro diamond ratios, grade or value be accompanied by an uncertainty figure obtained by statistical approaches.

Contact: JG Chapman, PO Box 45, Northbridge, Western

Australia 6865. chapman@gemdance.com 